14 VCE studies had findings compatible with Crohn's disease (NPV $75 \%$ ). All patients with positive findings of Crohn's disease on SB USS had evidence of SB Crohn's on VCE (PPV 100\%). Sub-analysis for Crohn's severity was carried out; of 11 VCE patients with moderate to severe Crohn's disease, 5 patients had a positive SB USS (sensitivity 45\%), however of 8 patients with mild Crohn's on VCE, no patients had a positive SB USS.

Conclusion SB USS has excellent positive predictive value (100\%) and specificity (100\%) for detection of SB Crohn's disease. All detected cases were moderate or severe identifying cases at higher risk of capsule retention. Sensitivity of SB USS is $26 \%$ rising to $45 \%$ in VCE proven moderate or severe disease. It follows that a positive expert SB USS in the context of suspected small bowel Crohn's is a definitive radiological result, on which therapy can be commenced. However, a negative SB USS should be followed by VCE or magnetic resonance enterography (MRE) if clinical suspicion remains.

Disclosure of Interest None Declared.

\section{PTH-181 BILE ACID MALABSORPTION: PREVALENCE COMPARABLE TO COELIAC DISEASE IN PATIENTS WITH CHRONIC DIARRHOEA}

doi:10.1136/gutjnl-2013-304907.668

1."M Kurien, ' J McConnell, ' IJ S Leeds, 'D S Sanders. 'Department of Gastroenterology, Royal Hallamshire Hospital, Sheffield, UK

Introduction Chronic watery diarrhoea is a common referral, with a host of possible aetiologies. To help establish a diagnosis a number of international guidelines have been created, defining diagnostic pathways. Bile acid malabsorption (BAM) is a potential cause, with high prevalence previously demonstrated by our group in patients with Diarrhoea predominant Irritable Bowel Syndrome (D-IBS). ${ }^{1}$ This study determines the prevalence of BAM and other organic conditions in patients referred with chronic diarrhoea (Group A), with findings compared to our previously published D-IBS cohort (Group B).

Methods A total of 92 consecutive patients referred to a tertiary referral centre with chronic diarrhoea, defined as more than 3 loose or liquid bowel movements a day for at least 4 weeks were evaluated (Group A). Demographic data, subsequent investigations and diagnostic yields of these tests were collected. All patients underwent haematological, biochemical and immunological testing prior to subsequent investigations. Statistical analysis was performed using SPSS with Fisher's exact test used to compare categorical data.

Results Medical records were identified in 89 of the 92 patients referred (mean age 50 years, range 18-86 years). Of these patients, 23 $(26 \%)$ had an organic cause for their diarrhoea identified (Table 1), with 6 having dual pathology. Inflammatory bowel disease was the most prevalent condition identified, with the prevalence of BAM being comparable to that seen for coeliac disease $(p=0.72)$. When evaluating diagnostic yields for BAM in Groups A and B, prevalence was significantly higher in the D-IBS Cohort (42\% vs $6 \%, p<0.001)$.

Abstract PTH-181 Table 1 Final Diagnoses in those referred with chronic diarrhoea

\begin{tabular}{ll}
\hline Diagnosis (total $\mathbf{n}=\mathbf{8 9}$ ) & Patients (\%) \\
\hline Diarrhoea-predominant Irritable Bowel Syndrome & $43(48)$ \\
Functional diarrhoea & $28(31)$ \\
Inflammatory Bowel Disease & $8(9)$ \\
Bile Acid Malabsorption & $5(6)$ \\
Lactose intolerance & $4(4)$ \\
Coeliac & $3(3)$ \\
Lymphocytic Colitis & $2(2)$ \\
Pancreatic Insufficiency & $1(1)$ \\
Small Bowel Bacterial Overgrowth & $1(1)$ \\
\hline
\end{tabular}

Conclusion In this study organic causes for chronic diarrhoea were identified in $26 \%$. Given that BAM had similar prevalence to coeliac disease in patients with chronic diarrhoea, we would advocate BAM investigations early within the diagnostic pathway.

Disclosure of Interest None Declared.

\section{REFERENCE}

Kurien $\mathrm{M}$ et al. Bile acid malabsorption: an under-investigated differential diagnosis in patients presenting with diarrhoea predominant irritable bowel syndrome type symptoms. Scand J Gastroenterol. 2011 Jul; 46(7-8):818-22

\section{PTH-182 WHAT IS THE DIAGNOSTIC YIELD OF DUODENAL BIOPSY AT UPPER GI ENDOSCOPY?}

doi:10.1136/gutjnl-2013-304907.669

'F Debonera, 'P Nair, 1,"M Thoufeeq. 'Endoscopy/Gastroenterology, Peterborough and Stamford Hospitals NHS Foundation Trust, Peterborough, UK

Introduction By and the large, the most common indication for obtaining biopsy of the 2nd part of the duodenum is in the diagnosis of coeliac disease. We wanted to identify the diagnostic yield of duodenal biopsies at upper GI endoscopy.

Methods We obtained clinical details of 500 patients who had duodenal biopsies taken at upper GI endoscopy (UGIE). This was provided by our local histo-pathology department. These included patients who had UGIE from October 2011 till September 2012.

We excluded indications related to coeliac follow up and suspected malignancy $(n=31)$. We collected data that included age of the patient, indication of the biopsy, findings at endoscopy and histology. We also looked at our results server to see if TTGs were sent for these biopsies.

Results Duodenal biopsies were taken to rule out coeliac disease in the context of a variety of symptoms: mainly iron-deficiency or other unspecified anaemia, but also chronic or intermittent diarrhoea, unexplained GI symptoms including nausea and vomiting, fatigue, abdominal pain and distension and weight loss.

Of the 469 biopsies, $89 \%(n=416)$ were reported as normal at histology and $11 \%(n=53)$ were reported as being abnormal.

$84 \%(n=45)$ of the abnormal biopsies were non specific in nature. Only $11.3 \%(n=6)$ of the abnormal biopsies were diagnosed as coeliac. A further 2 patients were diagnosed as coeliac disease after further clinical evaluation.

Of the 469 samples, 34\% ( $n=161)$ of patients had TTG serology was sent prior to biopsy. Only $50 \%(n=3)$ of those diagnosed with coeliac disease in this study had TTG done prior to biopsy.

$1.7 \%(n=8)$ of patients who had duodenal biopsies had a conclusive diagnosis of coeliac disease.

Only $0.4 \%(n=2)$ of patients who were TTG negative were diagnosed with coeliac disease based on histology and clinical correlation. There were no falsely positive TTG.

Conclusion The diagnostic yield of duodenal biopsies is low. It may be more cost-effective if we limit biopsies after being guided by tissue transglutamiase (TTG)

Disclosure of Interest None Declared.

\section{PTH-183 THE DIAGNOSTIC UTILITY OF ENDOSCOPIC DUODENAL BIOPSIES FOR GASTROINTESTINAL INVESTIGATION}

doi:10.1136/gutjnl-2013-304907.670

1,"N Patel, ' $V$ J Blackwell, 'P Patel, ${ }^{2} \mathrm{M}$ M Walker, 'H R Williams. 'Gastroenterology \& Hepatology, Imperial College London, London, UK; ${ }^{2}$ Medicine \& Public Health, University of Newcastle, Callaghan, Australia

Introduction Duodenal biopsies are usually taken at upper gastrointestinal endoscopy to exclude coeliac disease (CD). To date, few studies have investigated overall duodenal pathologies in this group. Serological testing for anti-tissue transglutaminase 
antibodies (TTG) should be performed in patients with a diagnosis of lymphocytic duodenosis (LD), which may herald CD. The main objectives of this study were to determine the indications and histological findings for duodenal biopsies in a teaching hospital setting, and the prevalence of $\mathrm{LD}$ and new diagnoses of CD in this cohort.

Methods 1043 patients with duodenal biopsies taken during upper gastrointestinal endoscopy in a teaching hospital setting in 24 months (2009-11) were studied retrospectively. Demographics, indications and histological findings were documented. Patients with known CD were excluded from the study; if an individual had more than one biopsy in the time period then only the first biopsy was included. When a specific abnormality was targeted for biopsy this was recorded. For LD patients, TTG results were recorded where available.

Results The patients' median age was 60 (range 16-97); 55\% were female. Anaemia was the commonest indication for biopsy (in 51\% of patients) followed by weight loss and abdominal pain.

$76 \%$ of biopsies were normal. LD was found in 155 patients (15\%), of whom $64 \%$ were female. Biopsy appearances of CD with positive TTG were found in 13 patients (1.2\%). Documentation of serology was not always available and so the incidence of CD in this population may be underestimated. Crohn's disease was diagnosed in 3 biopsies (0.29\%). Graft versus host disease, drug effect, lymphangiectasia and infection were seen (1 Giardia, 1 atypical mycobacteria, 1 Strongyloides).

70 duodenal biopsies (7\%) targeted specific abnormalities, including 6 malignancies ( 3 adenocarcinoma, 1 carcinoid tumour and 1 lymphoma) and 6 benign polyps including 2 adenomas. Of the 973 non-targeted biopsies, 772 (79\%) were normal.

There were no statistical differences between positive biopsy results and specific indications.

Conclusion Whilst the majority of biopsies were normal, significant abnormalities were found in $24 \%$ and $\mathrm{LD}$ was present in $15 \%$. While important for the diagnosis of $\mathrm{CD}$, duodenal biopsy was also vital for the diagnosis other pathologies including infection, Crohn's disease, drug effect and tumours.

Disclosure of Interest None Declared.

\section{PTH-184 PUSH ENTEROSCOPY LEADS TO A CHANGE IN DIAGNOSIS IN THE MAJORITY OF PATIENTS WITH POSITIVE COELIAC SEROLOGY AND NEGATIVE DUODENAL BIOPSY}

doi:10.1136/gutjnl-2013-304907.671

'R Willington, ${ }^{2} \mathrm{~V}$ Lashmar, ${ }^{3} \mathrm{~K}$ Benes, ${ }^{2} \mathrm{~K}$ Barnett, ${ }^{2} \mathrm{~S}$ Weaver, ${ }^{2} \mathrm{R}$ McCrudden, ${ }^{4} \mathrm{~T}$ Smith, ${ }^{2} \mathrm{C}$ Gordon, 5,6, ${ }^{*} \mathrm{P}$ Cilclitira, ${ }^{2,6} \mathrm{~S}$ McLaughlin. ${ }^{1} \mathrm{AMU}$, Southampton General Hospital, Southampton; ${ }^{2}$ Department of Gastroenterology; ${ }^{3}$ Department of Pathology, Royal Bournemouth Hospital, Bournemouth; ' $D$ Department of Gastroenterology, Southampton General Hospital, Southampton; ${ }^{5}$ Department of Gastroenterology, St Thomas' Hospital; ${ }^{6}$ Diabetes and Nutritional Sciences Division, King's College London, London, UK

Introduction Coeliac disease is associated with significant morbidity, and is an underdiagnosed condition with an estimated prevalence of $1 \%$ in the UK population. Current British Society of Gastroenterology Society guidelines classify individuals with positive coeliac serology and negative duodenal biopsy to latent coeliac disease and do not recommend further investigation or the initiation of a gluten free diet.

In our recent practise patients at our institution with a strong clinical suspicion of coeliac disease and negative duodenal biopsies following a gluten containing diet undergo a push enteroscopy with biopsies of the jejunum and duodenum and histological reassessment of the original duodenal biopsies before a diagnosis of latent coeliac disease is made.

We aimed to investigate whether push enteroscopy and histological reassessment increased the diagnostic yield of coeliac disease.
Methods We searched our prospective endoscopy database for all patients with positive EMA or TTG results but negative D2 biopsies, who had undergone enteroscopy for a possible diagnosis of coeliac disease since 2007. Data including serology, result of histological reassessment of duodenal biopsies and assessment of jejunal biopsies were recorded.

Results 13 patients were identified; 4 (31\%) were male, the median age was 45 (range 19-78). In all 13, EMA testing had been performed, of which 9 were positive $(69 \%)$. In 9 , TTG results were available, of which 9 were positive (100\%). Following review of the original duodenal biopsies by a second pathologist, 7 (54\%) of the 13 patients were re-classified to active coeliac disease; median Marsh grade of 1 (range $0-2$ )

Of the remaining 6 patients, 5 (83\%) had evidence of active coeliac disease on jejunal biopsy; median Marsh grade of 2 (range 0-3b). Review of the jejunal biopsies alone, without review of original duodenal biopsies, would have led to active coeliac disease being diagnosed in 12 (92\%) of 13 patients.

Conclusion In this small retrospective series of patients with positive coeliac serology and negative duodenal biopsies, repeat histological assessment and jejunal biopsy led to a change in diagnosis in $92 \%$ of patients. These findings are unlikely to be limited to our institution. Our findings support the recommendation that a diagnosis of latent coeliac disease should only be made following repeat histological assessment and enteroscopy with jejunal biopsy.

Disclosure of Interest None Declared.

\section{PTH-185 CAPSULE ENDOSCOPY AND OBSCURE GASTROINTESTINAL BLEEDING: EXPERIENCE FROM A TERTIARY CENTRE}

doi:10.1136/gutjnl-2013-304907.672

1."P S Sidhu, 'K Drew, 'M E McAlindon, ${ }^{1} \mathrm{R}$ Sidhu. 'Gastroenterology, Royal Hallamshire Hospital, Sheffield Teaching Hospitals, Sheffield, UK

Introduction Capsule endoscopy (CE) is the first line modality to investigate the small bowel (SB) in patients with obscure gastrointestinal bleeding (OGB). We investigated the largest cohort of patients to date with OGB who underwent CE.

Methods All patients referred for CE for OGB were included retrospectively. Data was collected for demographics, clinical indication (overt bleeding $(\mathrm{OB})$ or iron deficiency anaemia (IDA)) the presence of co-morbidity, medications including anticoagulation, diagnostic yield (DY) and subsequent follow up.

Results A total of 1324 patients were identified. The mean age was 60 years with females consisting of $55 \%$. The indication for CE was IDA in $73 \%(n=971)$ and OB in $27 \%$. Significant co-morbidities were present in $38 \%$ of patients. Whilst $8 \%$ of patients were on warfarin, $6 \%(n=84)$ were transfusion dependent. The cumulative number of lesions identified was 1086.The overall DY was $36 \%(n=473)$ for lesions deemed to be the source of OGB. On logistic regression, increasing age $(p<0.001)$ and transfusion dependence $(p=0.02)$ were associated with a DY with CE. The commonest diagnosis was angioectasia (AE) in $23 \%(n=302)$. Other SB findings included ulcers and erosions in $25 \%(\mathrm{n}=326)$, fresh blood without a definite source in $6 \%(\mathrm{n}=79)$ whilst tumours, polyps and crohns disease represented $2 \%$ each respectively. Miscellaneous findings in the SB included diverticulae (14), SB strictures (15), SB varices (9), dielaufoy (1) and others (28). There was no significant difference in the DY between those with IDA and $O B(p=0.35)$ and between the sexes $(p=0.44)$. Management was altered in $78 \%(n=367)$ of those with a positive DY, in the form of further procedures $(42 \%, n=153)$. Of these, $35 \%(n=53)$ had double balloon enteroscopy, $24 \%(n=36)$ had push enteroscopy, $23 \%(n=35)$ had repeat standard endoscopy, 17 patients were referred for surgery whilst 2 patients had intraoperative enteroscopy. $34 \%(n=52)$ of patients received argon 